\title{
Can the pilocarpine phenylephrine provocative test be used to detect covert angle closure?
}

Glaucoma Unit, St Paul's Eye Hospital, Old Hall Street, Liverpool L3 9PF P K Wishart

Correspondence to:

Mr P K Wishart, FRCS Glas.

Accepted for publication

2 April 1991

\begin{abstract}
Mapstone introduced the term 'partial angle closure' to describe how eyes with apparently open angles could be damaged by a covert angle closing mechanism, identifiable by a positive result of the pilocarpine phenylephrine provocative test (PPPT). This paper presents a 10-year follow-up of 68 patients with narrow but open anterior chamber angles who, on the basis of a positive PPPT, underwent peripheral iridectomy (PI) or laser iridotomy LI). In the 42 patients with glaucoma or ocular hypertension the mean intraocular pressure (IOP) was unchanged following iridectomy; $45 \%$ of eyes which showed glaucomatous optic disc damage at presentation and $25.6 \%$ of ocular hypertensive eyes subsequently required trabeculectomy to achieve IOP control. In 16 patients with no ocular abnormality other than a narrow angle 19 eyes had positive PPPTs and underwent PI. After a 10year follow-up only one of the 13 untreated fellow eyes developed angle closure glaucoma. As this is usually bilateral, many of the positive tests in this group of patients may therefore have been false positives. This study shows that eyes with narrow angles which had positive PPPT results did not benefit from PI iridectomy or LI and therefore a positive PPPT is not indicative of the presence of an angle closing mechanism.
\end{abstract}

To identify partial closure of the anterior chamber angle ${ }^{l}$ by a covert angle closing mechanism - an event leading to ocular hypertension and some cases of open angle glaucoma unless relieved by a peripheral iridectomy $(\mathrm{PI})^{2-4}$ - Mapstone advocated the use of the pilocarpine phenylephrine provocative test (PPPT). ${ }^{2-4}$ The difficulty of predicting the development of angle closure glaucoma (ACG) in an eye with a narrow anterior chamber (AC) angle ${ }^{5}$ could be overcome, Mapstone believed, by use of the PPPT, as a positive result identifies the presence of an angle closing mechanism. ${ }^{67} \mathrm{He}$ described the PPPT as more helpful than gonioscopy in the management of eyes with narrow angles, ${ }^{6-9}$ and an eye with a positive result of the test should, he stated, undergo a PI to relieve the partial angle closure, lower the raised IOP, and prevent further outflow damage. ${ }^{24}$

This study reports an average 10-year followup of asymptomatic patients with shallow anterior chambers, narrow but open angles, and raised IOP who underwent a PI or LI when a positive result to the PPPT was taken as an indication that partial angle-closure was present. Follow-up of a group of patients with otherwise normal eyes but with narrow angles who had positive PPPTs and underwent PI is also presented.

\section{Patients and methods}

Between 1975 and 1986 asymptomatic patients with narrow angles (angle width van Herick grade 2 or less ${ }^{10}$ and shallow anterior chamber depths (measured by Haag-Streit pachymetry) with or without glaucomatous optic disc damage attending the Glaucoma Unit at St Paul's Eye Hospital underwent a PPPT to detect if covert angle closure was present. The IOP was above $21 \mathrm{~mm} \mathrm{Hg}$ in most of them. In all cases presentation was due to the incidental finding of a narrow angle and/or raised IOP and/or glaucomatous disc damage. In no case was there a history suggestive of a previous attack of acute or subacute ACG.

A positive result was taken as a rise of 8 $\mathrm{mm} \mathrm{Hg}$ in association with a closed angle. Mapstone did not rely on gonioscopy to show if angle closure was present during a PPPT, but assessed this by examining the four quadrants of the eye by van Herick and Shaffer's method ${ }^{10}$ to detect peripheral iridocorneal contact. ${ }^{6}$ If positive, a PI or LI was performed. If the PPPT gave a negative result, the patient was followed up and treated medically, with repetition of the PPPT at yearly intervals.

Follow-up examination included repeat measurement of the axial AC depth, measurement of the IOP by Goldmann applanation tonometry, stereoscopic optic disc examination performed with the slit-lamp biomicroscope, and Zeiss four-mirror indentation gonioscopy. Visual field analysis was performed with the Friedmann and, later, with the Humphrey visual field analyser to confirm glaucomatous visual field loss when the patient's vision permitted.

\section{Results}

One hundred and thirty-six patients with narrow angles, belonging to one of three groups on clinical grounds, underwent PPP testing:

A. Glaucomatous. Patients with glaucomatous optic disc cupping in one or both eyes, raised IOP, and narrow angles.

B. Ocular hypertension. Patients with raised IOP, normal optic discs, and narrow angles.

C. Normals. Patients with normal IOP, normal optic discs, and narrow angles.

Nine patients were lost to follow-up or had died by 1987 , and they were therefore excluded from the study.

Table 1 shows the number of patients and eyes with positive PPPT results and the average age and length of follow-up. Table 2 shows the mean IOP at presentation, and mean anterior chamber 
Table 1 Number of patients and number of eyes with positive PPPTs. Age of patients in years at presentation with years of follow-up in each group - mean (with standard deviation)

\begin{tabular}{lllll}
\hline Group & Patients & $\begin{array}{l}\text { No eyes } \\
\text { +vePPPT }\end{array}$ & Age $(S D)$ & $\begin{array}{l}\text { Follow-up } \\
\text { years }(S D)\end{array}$ \\
\hline A & 20 & 30 & $66 \cdot 2(11.0)$ & $11.95(3.07)$ \\
B & 32 & 43 & $63.45(8.13)$ & $8.94(2.96)$ \\
C & 16 & 19 & $61.38(8.82)$ & $10.0(2.66)$ \\
\hline
\end{tabular}

Table 2 Intraocular pressure at presentation, and axial anterior chamber depth of eyes with positive PPPTs in each group - mean (standard deviation)

\begin{tabular}{lll}
\hline Group & $A C D, m m(S D)$ & $I O P, m m ~ H g(S D)$ \\
\hline A & $2 \cdot 14(0.29)$ & $34 \cdot 2(11 \cdot 1)$ \\
B & $1.96(0.25)$ & $28 \cdot 2(4 \cdot 4)$ \\
C & $2.03(0.55)$ & $18 \cdot 29(2 \cdot 0)$ \\
\hline
\end{tabular}

depth in the three groups. There was no difference (within the error of method) between the AC depths at the time of presentation and the follow-up measurements which appear in Table 2. In 10 eyes $(9 \cdot 5 \%)$ there was no record of the AC depth. The results are given as mean with standard deviation (SD).

GROUP A: GLAUCOMATOUS

Twenty patients with glaucomatous damage to one or both optic discs, raised IOP, narrow angles, and shallow anterior chambers had positive PPPT results in one or both eyes. Of the 20 patients ( 40 eyes) 10 were affected bilaterally and 10 unilaterally. The excluded eyes comprise eight eyes with negative PPPT results and two eyes which had positive PPPT results but were normotensive with normal optic discs.

Thirty eyes had glaucomatous disc damage, raised IOP, a narrow anterior chamber angle, and visual field loss on Friedmann field analysis. As a consequence of a positive result to the PPPT these eyes underwent peripheral iridectomy.

The mean IOP at presentation was $34 \cdot 2$ (SD $11 \cdot 1) \mathrm{mm} \mathrm{Hg}$ and had been reduced to $23 \cdot 2$ (SD 6.9) $\mathrm{mm} \mathrm{Hg}$ with medical treatment immediately prior to PI. The mean IOP at approximately two months after iridectomy, was $28 \cdot 8$ (SD 5.59) $\mathrm{mm} \mathrm{Hg}$.

Despite the PI, the IOP was only lowered in one eye postoperatively. Thirteen $(45 \%)$ of the remaining 29 eyes in this group ultimately required trabeculectomy to lower the IOP, the others requiring the same or increased medical treatment postoperatively as preoperatively. Thirteen eyes developed cataracts and 12 underwent extracapsular cataract extraction and lens implant (ECCE $+\mathrm{IOL})$. Gonioscopy during follow-up showed open angles. Visual loss due to glaucomatous disc damage was marked in 18 patients, and only one patient (two eyes) retained a visual acuity (VA) of $6 / 9$ with only moderate damage to the visual field. In 15 eyes the final visual acuity was between no light perception and $6 / 24$ owing to glaucomatous damage to central vision.

GROUP B: OCULAR HYPERTENSION

Ninety patients with ocular hypertension and narrow anterior chamber angles underwent PPP testing. Fifty-eight had negative PPPT results in both eyes, and therefore the outcome in them is not included in this study but has been presented elsewhere. ${ }^{11}$ The remaining 32 patients (64 eyes) with positive PPPT results are presented here. All eyes in this group had a recorded pretreatment IOP of at least $23 \mathrm{~mm} \mathrm{Hg}$ in one or both eyes and normal optic discs. One eye was thought to have peripheral anterior synechiae of the angle, but postoperative gonioscopy did not confirm this finding. Of the 32 patients with positive PPPT results 11 were positive bilaterally, and 21 unilaterally, giving 43 eyes with positive results. These 43 eyes underwent iridectomy 33 peripheral iridectomy and 10 argon laser iridotomy.

The mean preoperative IOP was 27.66 (SD $4 \cdot 18) \mathrm{mm} \mathrm{Hg}$ for 39 eyes on no medical treatment and four eyes on treatment with timolol or pilocarpine. The mean postoperative IOP at approximately two months following PI or LI was 28.02 (SD 5.8 ) $\mathrm{mm} \mathrm{Hg}$, with two eyes requiring medication and 41 on no treatment. Four eyes became normotensive after PI with an average fall in IOP of $7 \mathrm{~mm} \mathrm{Hg}$, though after five years of follow-up three became hypertensive again. Three others, after iridectomy, had a fall in IOP (average $4 \mathrm{~mm} \mathrm{Hg}$ ), though they remained hypertensive. In the remaining 36 the IOP was not lowered and was treated medically. Gonioscopy showed open angles in all eyes. Eleven eyes $(25 \cdot 6 \%)$ subsequently required trabeculectomy for IOP control, and nine developed cataracts requiring ECCE + IOL. Six eyes developed optic disc changes over the follow-up period; three eyes showed glaucomatous optic disc cupping, and three optic discs were damaged by vascular occlusions. Twenty two eyes retained vision of $6 / 9$ or better, five had a reduction of visual acuity due to mild lens opacity, and two due to diabetic maculopathy.

\section{GROUP C: NORMALS}

Sixteen patients (32 eyes) with no detectable abnormality other than an incidental finding of a narrow anterior chamber angle had PPP testing. Fifteen patients had positive PPPT results in one eye, and one patient had a positive result in both. Seventeen eyes therefore underwent peripheral iridectomy after initial presentation. The fellow eye in each group was followed up in the Glaucoma Unit with repeat PPP testing. Two fellow eyes later became positive to PPPT and underwent laser iridotomy. The fellow eye in one patient, despite negative PPPT results, spontaneously developed an acute attack of angle closure glaucoma. The anterior chamber depths in this patient were $1.1 \mathrm{~mm}$ and $1.3 \mathrm{~mm}$, and the initial PPPT on the first eye induced an attack of acute angle closure glaucoma (ACG). In the remaining 12 patients the 12 fellow eyes retained open anterior chamber angles without pathology and with no signs or symptoms of angle closure glaucoma after an average 10-year follow-up. Four of the initially normotensive eyes that underwent PI later developed a raised IOP but retained normal optic discs. By the end of the follow-up period eight eyes had developed dense cataracts and four underwent ECCE+IOL. 
Table 3 Results of surgery (PI/LI) on eyes in groups $A, B, C$ - mean IOP in $\mathrm{mm} \mathrm{Hg}(S D)$

\begin{tabular}{llll}
\hline & Group A & Group B & Group C \\
\hline Mean IOP pre-PI & $23 \cdot 2(6 \cdot 9)$ & $27 \cdot 66(4 \cdot 18)$ & $18 \cdot 29(2 \cdot 0)$ \\
Mean IOP post-PI & $28 \cdot 8(5 \cdot 59)$ & $28 \cdot 02(5 \cdot 8)$ & $19 \cdot 47(3 \cdot 35)$ \\
Required trabeculectomy & $45 \%$ & $25 \cdot 6 \%$ & Nil \\
Developed cataract & $43 \%$ & $21 \%$ & $36 \cdot 8 \%$ \\
\hline
\end{tabular}

Seven of these cataracts developed in eyes that had undergone peripheral iridectomy, and one untreated eye developed a cataract.

Table 3 summarises the results of the three groups undergoing surgery.

\section{Discussion}

Acute, subacute, and chronic angle closure glaucoma are precise and well established terms for varieties of pupillary block glaucoma diagnosed by a combination of history and clinical examination including gonioscopy. ${ }^{12-15}$ Mapstone has introduced the term partial angle

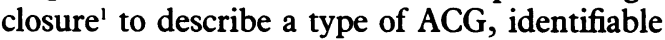
by a PPPT and responding to a peripheral iridectomy, ${ }^{34}$ which can occur without symptoms in eyes with apparently open angles.

Clinical experience has shown peripheral iridectomy to be a highly successful treatment for acute ACG, with, on average, $70 \%$ of eyes with acute ACG achieving normal IOP following PI alone. ${ }^{16-20}$. Similarly, eyes with subacute ACG respond to a PI. ${ }^{192}$ or $\mathrm{LI}^{22}$ but often in addition require topical medication.

Studies of patients with medically uncontrolled chronic angle closure glaucoma have shown that even where field loss and significant synechial closure of the anterior chamber angle is present approximately $70 \%$ of eyes will achieve satisfactory control with PI of LI and topical medication. ${ }^{19-24}$ In eyes suffering from chronic angle closure glaucoma without field loss and without peripheral anterior synechiae in the angle PI is strikingly successful, with $66 \%$ of such eyes in Gelber and Anderson's study, ${ }^{23} 69 \%$ in Playfair and Watson's series, ${ }^{21}$ and $78 \%$ in Forbes's study ${ }^{14}$ cured by PI alone.

In contrast to the marked success of peripheral iridectomy in the treatment of angle closure glaucoma in the above studies, it failed to help the patients in groups A and B of this study, in whom a positive PPPT result was said to have identified partial angle closure as the underlying cause of their raised IOP. Only one of the eyes in group A seemed to benefit from PI, and $50 \%$ of eyes in this group suffered severe progressive visual loss from glaucoma. $45 \%$ of eyes in group A ultimately required trabeculectomy. The outcome of the eyes in group B which were diagnosed as having partial angle closure showed that after PI only four $(9 \cdot 3 \%)$ became normotensive off medication, and this fall was not sustained in three eyes. Had the eyes in group B been suffering from chronic ACG, they would have belonged to the group with the most favourable prognosis, having neither field loss nor PAS, and at least $66 \%$ would be expected to become normotensive following PI alone..$^{142123}$ $25.6 \%$ of eyes in group B ultimately required trabeculectomy to achieve IOP control.
In the normotensive patients in group $C$ the need for an iridectomy cannot be judged postoperatively on the basis of a change in IOP. However, as angle closure glaucoma is usually a bilateral disease, occurring in some $70 \%$ of fellow eyes, ${ }^{25-28}$ the fate of the fellow eyes in group $\mathrm{C}$ can give some indication as to whether this group was genuinely suffering from covert ACG. Only one (8\%) of the fellow eyes in this group without an iridectomy developed ACG, and this was in a high risk eye with an exceptionally shallow anterior chamber depth $(1 \cdot 1 \mathrm{~mm}) .^{25}$ The other fellow eyes in this group showed no signs or symptoms of ACG and retained narrow though open angles throughout the 10-year follow-up.

The only indication that the patients in this study were suffering from angle closure was a positive PPPT result, which Mapstone believed identified partial angle closure or a covert angle closing mechanism. ${ }^{1-4}$ The failure of PI to benefit the eyes in groups A and B leads one to conclude that the patients in groups A and B could not have been suffering from ACG. Similarly, followup of the patients in group $C$ shows that, with the exception of one eye, this group was not at high risk of angle closure glaucoma.

Mapstone's PPPT relied on van Herick and Shaffer's method ${ }^{10}$ of estimation of the depth of the peripheral anterior chamber to determine when the angle was closed ${ }^{136}$ and not on gonioscopy, which he considered provided only a subjective assessment of narrowness of the anterior chamber angle. ${ }^{1467}$. This study has shown that a large number of eyes with narrow angles developed an $8 \mathrm{~mm} \mathrm{Hg}$ rise in IOP and a closed angle assessed by van Herick and Shaffer's method - a positive result - on PPP testing. As follow-up has not shown these eyes to have been suffering from ACG, it appears likely that the unphysiological maximal sympathetic and parasympathetic forces occurring simultaneously during the PPPT resulted in the creation of a pupillary block force that led to shallowing of the periphery of the anterior chamber and a rise of pressure. As $100 \%$ of eyes in group A with glaucomatous optic discs had positive results to PPP testing and in group B only $24 \%$ of eyes ( 43 of 180 tested) gave positive test results, this may be an indication that an already damaged outflow cannot cope with the alteration in fluid dynamics occurring in an eye during a PPPT. This confirms the findings of a recent study which showed that eyes with a history of subacute angle closure glaucoma were 10 times as likely to have a positive PPPT result if they showed optic disc damage than if the optic disc was normal. ${ }^{17}$

It is evident from the results of ths study that an eye with a narrow angle can develop an $8 \mathrm{~mm} \mathrm{Hg}$ rise in IOP and peripheral iridocorneal contact when subjected to a PPPT yet not be suffering from, or at risk of, angle closure glaucoma. If an eye with a narrow angle, raised IOP, and glaucomatous optic disc damage undergoes a PPPT, a positive result is almost inevitable, irrespective of the aetiology of the glaucoma. The belief that the PPPT detects angle closure glaucoma is therefore erroneous, and the evidence that a positive PPPT has identified an otherwise undescribed form of angle closure - 
partial angle closure - is not substantiated. Use of the PPPT is therefore not recommended in clinical practice.

I would like to thank Mrs C Owen for technical and administrative help.

1 Mapstone R. Partial angle closure. Br f Ophthalmol 1977; 61: $525-30$

2 Mapstone $R$. Mechanisms in ocular hypertension. BrF Ophthalmol 1979; 63: 325-30.

3 Mapstone R. Mechanisms in open angle glaucoma. Brf Ophthalmol 1978; 62: 275-82.

4 Mapstone R. Narrow angle glaucoma. Doc Ophthalmol Proc Ser 1980; 22: 185-93.

5 Spaeth GL. Gonioscopy: uses old and new. The inheritance of occludable angles. Ophthalmology 1978; 85; 222-31.

6 Mapstone R. Provocative tests in closed-angle glaucoma. BrF Ophthalmol 1976; 60: 115-9.

7 Mapstone R. Acute shallowing of the anterior chamber. Br F Ophthalmol 1981; 65: 446-51.

8 Mapstone R. One gonioscopic fallacy. Brf Ophthalmol 1979; 63: $221-4$.

9 Mapstone R. The fellow eye. Br f Ophthalmol 1981; 65: 410-3.

10 van Herick W, Shaffer RN. Estimation of width of angle of anterior chamber. Incidence and significance of the narrow anterior chamber. Incidence and significance

11 Batterbury $M$, Wishart PK. Is a narrow angle a specific risk factor in ocular hypertension. Report presented at the Glaucoma Group meeting, London 1990 .

12 Shields MB. Primary angle-closure. Textbook of glaucoma. Baltimore: Williams and Wilkins, 1987: 165-85.

13 Spaeth GL. The normal development of the human anterior chamber angle: a new system of descriptive grading. Trans Ophthalmol Soc UK 1971; 91: 709-39.

14 Forbes $M$. Indentation gonioscopy and efficacy of iridectomy in angle-closure glaucoma. Trans Am Ophthalmol Soc 1974; $488-515$.
15 Campbell DG. A comparison of diagnostic techniques in angle-closure glaucoma. Am $f$ Ophthalmol 1979; 88: angle-closur.

16 Playfair TJ, Watson PG. Management of acute primary angleclosure glaucoma: a long-term follow-up of the results of peripheral iridectomy used as an initial procedure Br F Ophthalmol 1979; 63: 17-22.

17 Wishart PK. Does the pilocarpine phenylephrine provocative test help in the management of patients with acute and subacute angle-closure glaucoma? Brf Ophthalmol 1991; 75: $284-7$.

18 David R, Tessler Z, Yassur Y. Long-term outcome of primary acute angle closure glaucoma. Br f Ophthalmol 1985; 69: $261-2$.

19 Murphy MB, Spaeth GL. Iridectomy in primary angle-closure glaucoma. Arch Ophthalmol 1974; 91: 114-22.

20 Lowe RF. Primary angle-closure A review 5 years after bilateral surgery. Br f Ophthalmol 1973; 57: 457-63.

21 Playfair TJ, Watson PG Management of chronic or intermittent primary angle-closure glaucoma: a long-term followup of the results of peripheral iridectomy used as an initial procedure. Br F Ophthalmol 1979; 63: 23-8.

$22 \mathrm{McG}$ alliard JN, Wishart PK. The effect of Nd:YAG laser iridotomy on intraocular pressures in hypertensive eyes with shallow anterior chambers. Eye $1990 ; 4: 823-9$.

23 Gelber EC, Anderson DR. Surgical decisions in chronic angleclosure glaucoma. Arch Ophthalmol 1976; 94: 1481-4.

24 Gieser DK, Wilensky JT. Laser iridectomy in the management of chronic angle-closure glaucoma. Am 7 Ophthalmol ment of chronic

25 Lowe RF. The natural history and treatment of primary angleclosure glaucoma. Am $\mathcal{F}$ Ophthalmol 1966; 61: 642-51.

26 Edwards RS. Behaviour of the fellow eye in acute angle-closure glaucoma. Brf Ophthalmol 1982; 66: 576-9.

27 Lowe RF. Acute angle-closure glaucoma. The second eye: an analysis of 200 cases. Br F Ophthalmol 1962; 46: 641-50.

28 Hyams SW, Friedman Z, Keroub C. Fellow eye in angleclosure glaucoma. Brf Ophthalmol 1975; 59: 207-10. 